\title{
Collagen changes in the pathogenesis of systemic sclerosis
}

\author{
MALCOLM I. V. JAYSON \\ From the Department of Medicine, University of Bristol, and Royal National Hospital for \\ Rheumatic Diseases, Bath
}

Progressive systemic sclerosis (PSS) is a multisystem disorder. The prevalence of different forms of systemic involvement as determined by Campbell and LeRoy (1975) is shown in the Table. As it may be difficult to detect early and mild organ involvement the true prevalence of several of the manifestations is probably under estimated. Such changes are due to a combination of widespread vascular disease, fibrosis, and atrophy.

Table Systemic involvement in progressive systemic sclerosis (Campbell and LeRoy, 1975)

\begin{tabular}{lll} 
System & & Incidence $(\%)$ \\
\cline { 1 - 1 } Raynaud's phenomenon & & 78 \\
Skin & 90 \\
Oesophagus & & 52 \\
Small and large bowel & & 15 \\
Lung & 43 \\
Pericardium & 11 \\
Heart & 40 \\
Hypertension & 21 \\
Kidney & 35 \\
Articular & 25 \\
Muscle & 20 \\
Anaemia & 27
\end{tabular}

\section{Collagen proliferation}

Pathological examination of most organs reveals fibrous replacement of the tissues. However, early biochemical studies (Fleischmajer, 1964) failed to show excess collagen in the skin. Moreover, $x$-ray diffraction and amino-acid analysis (Fleischmajer, 1964; Neldner et al., 1966) failed to show any difference between collagens from normal and PSS skins. However, Hayes and Rodnan (1971) and Fleischmajer et al. (1972) showed by electron microscopy that PSS collagen contains an abnormal number of fine immature collagen fibrils with incomplete crossbanding and beaded filaments resembling fetal skin collagen, implying that it is recently formed.

Newly formed collagen is comparatively soluble and can be readily extracted. Uitto et al. (1971) demonstrated increased solubility of collagen in scleroderma skin. In newly formed collagen reducible cross-links are readily detected, whereas when the collagen matures they become stabilized and the proportion detectable falls. Herbert et al. (1974) demonstrated excess reducible cross-links in the skin of patients with systemic sclerosis indicating proliferation of new collagen. Biopsy of the centre and of the growing edge of a plaque of morphoea showed more new collagen at the edge than at the centre. Another index of the rate of collagen synthesis is obtained by determining the activity of the enzyme protocollagen proline hydroxylase. Raised levels have been found in scleroderma skin (Uitto et al., 1969). Indeed, Keiser et al. (1971) found raised levels in clinically unaffected skin of PSS patients although the levels were higher still where the disease was clinically manifest.

Rates of collagen synthesis have been measured more directly by culturing scleroderma tissue in a medium containing radioactive-labelled proline and measuring the rate of synthesis of hydroxyproline, an amino-acid unique to collagen. Keiser and Sjoerdsma (1969) reported raised synthesis rates in tissue culture of human skin, but this was not confirmed by Uitto et al. (1970a). This discrepancy may reflect one of the major problems of tissue culture studies-that minor variations in technique or in the media may profoundly alter the results. However, LeRoy $(1972,1974)$ and Fleischmann and LeRoy (1975) cultured PSS skin fibroblasts and demonstrated increased synthesis of soluble collagen compared with control fibroblasts. This ability to synthesize excess collagen persisted for up to 15 subcultures of the original primary culture. The collagens formed by normal and PSS fibroblasts were identical, suggesting that the disease is due to altered regulation of the fibroblasts but with normal assembly of the collagen molecule. However, Kovacs and Fleischmajer (1974) described differing metabolic requirements for optimal growth of PSS and normal fibroblasts. When grown under standard culture conditions the cell doubling time of PSS fibroblasts is significantly lower than normal, but is restored to normal by enriching with additional nutrients.

\section{Immunological factors}

The association between systemic sclerosis and other connective tissue diseases is well recognized (for example, see Dubois et al., 1971). Indeed, Sharp et al. (1972) described patients with systemic lupus 
erythematosus, systemic sclerosis, polymyositis, and a specific antibody to an extractable nuclear antigen and thought this was a distinct entity-mixed connective tissue disease. These associations lead us to feel that immunological factors may play some part in the pathogenesis of systemic sclerosis.

There is conflicting evidence about altered cellular immunity in PSS (Winkelstein et al., 1972; Rowell, personal communication). Direct immunofluorescence has failed to show any changes in either normal or affected areas with the exception of low density immunofluorescent bands at the dermoepidermal junction in areas of telangiectasiae. Since identical changes occur in telangiectasiae due to other causes this is probably not significant (Jablonska et al., 1975). However, McGiven et al,. (1971), Scott and Rowell (1974), and Gerber (1975) demonstrated immunoglobulins and complement principally in the renal arterioles but also on occasions in the glomeruli of PSS patients. The changes resembled those of malignant hypertension. However, not all patients had this complication, so these workers suggested that immune complex deposition may precede malignant hypertension.

There is no consistent change in immunoglobulin levels in PSS (Spencer and Winkelmann, 1971). Antinuclear factor has been reported in up to $90 \%$ of patients with systemic sclerosis and rheumatoid factor in up to $35 \%$ (Rothfield and Rodnan, 1968; Clark et al., 1971; Jordan et al., 1971; Rodnan, 1972; Davis and Jayson, 1976). The antinuclear factor is usually of low titre and no correlation has been found between the extent or severity of the disease and the antinuclear activity (Rothfield and Rodnan, 1968). However, rheumatoid factor tends to occur in those with the highest antinuclear factor titres.

Ritchie (1970) found antinucleolar antibodies in $54 \%$ of patients with PSS, in $26 \%$ with systemic lupus erythematosus, and in $9 \%$ with rheumatoid arthritis. Alarcón-Segovia and Fishbein (1975) and Alarcón-Segovia et al. (1975) found antibodies to RNA in $\mathbf{4 0}$ out of $\mathbf{4 0}$ patients with PSS. These antibodies were specific to the uracil base of RNA and therefore for single-stranded RNA. In contrast, they found RNA antibodies in 20 out of 40 patients with systemic lupus erythematosus, but these were heterogenous, directed at various sites of the RNA molecule, and reacted with double-stranded RNA. They suggest that these uracil-specific, antisingle-stranded RNA antibodies may be the same as the antinucleolar antibodies found by Ritchie (1970). They may be directed against an RNA virus which might be responsible for the disease.

\section{Vascular factors}

Vascular hyperreactivity in the hands to cold (Raynaud's phenomenon) almost invariably ac- companies systemic sclerosis (Campbell and LeRoy, $\stackrel{3}{\square}$ 1975). In PSS it is always bilateral in contrast with occasionally being unilateral when local factors $\overrightarrow{\vec{F}}$ play some part in its pathogenesis (Velayos et al., $\stackrel{?}{+}$ 1971). This hyperreactivity has been documented by peripheral blood flow measurements (LeRoy et $\overline{\bar{\sigma}}$. al., 1971; Coffman and Cohen, 1971). The specific $\frac{\text { ? }}{\sigma}$ defects seem to be in capillary blood flow with $\propto$ selective vasoconstriction of the blood vessels that $\infty$ regulate heat exchange. Such vascular hyper- $\vec{\circ}$

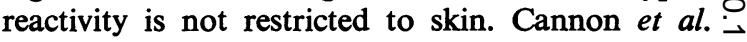
(1974) demonstrated reduction of renal blood flow $\vec{\omega}$ in PSS patients on induction of cutaneous Raynaud's $\frac{\sigma}{\circ}$ phenomenon by cooling, in contrast with the normal slight increase in renal blood flow. Trigeminal $\omega_{\infty}$ neuropathy, reported in association with PSS is (Ashworth and Tait, 1971), may be another example $\frac{\mathrm{C}}{0}$ of vascular hyperreactivity.

Morphological changes are found in the small $N$ blood vessels. Maricq and LeRoy (1973) described o the large dilated capillary loops in the nail beds of 웅 PSS patients, and Norton et al. (1968) showed on biopsy by electron microscopy of muscle tissue a Tr reduction in the number of capillaries to only about $20 \%$ of the normal. Those remaining appeared abnormal. There was an increased number of $₹$ venules. Similar but less severe changes were seen systemic lupus erythematosus.

Narrowing and obstruction of the arterioles and small arteries have been demonstrated by arteriography by Dabich et al. (1972). However, they could find only a poor correlation between the extent of the skin disease and the severity of the arterial disease. Sometimes obstruction of vessels antedated the skin disease and vice versa. Histological vascular abnormalities can be demonstrated in absence of sclerosis (Norton and Nardo, 1970) although the systemic nature of the disease usually reflects the systemic distribution of these lesions. The fact that normal skin in a PSS patient becomes sclerodermatous when transplanted into an affected site makes it more likely that local vascular factors play some part in the pathogenesis of the disease (Fries et al., 1971).

Characteristic histological changes are seen in the small arteries and arterioles in the kidneys, lungs, and other organs (D'Angelo et al., 1969; Trell and Lindström, 1971; Cannon et al., 1974). The vessels show concentric mucoid intimal proliferation which may obstruct their lumen. There may also be fibrosis of the adventitia. In the kidney there may be fibrinoid necrosis of the arterioles and glomerular changes which mimic the appearances in malignant hypertension. In the lungs the arterial lesions are typical of those of primary pulmonary hypertension. This is interesting because it is known that primary pulmonary hypertension may be associated with Raynaud's phenomenon. 
There is no evidence of sympathetic hyperactivity in the pathogenesis of the Raynaud's phenomenon (Fries, 1969; Sapira et al., 1972). Although peritoneal fibrosis has been reported in the carcinoid syndrome (Hallén, 1964) there is no evidence to suggest that this is relevant to PSS (Tuffanelli, 1963).

In the early stages of PSS inflammatory changes may be seen in the tissues (Rodnan and Medsger, 1966; Fleischmajer and Nedwich, 1972), particularly as cuffs of chronic inflammatory cells around the small arteries and arterioles (Campbell and LeRoy, 1975). They may be the result of the immune complex deposition described earlier. Johnson and Ziff (1974) suggested that lymphokine can stimulate the synthesis of collagen and so might lead to thickening of the vessel wall. Newly formed soluble collagen could diffuse through this wall into the lumen. One of the properties of collagen is that it binds platelets and could act as an initiating factor in intimal damage and vascular thrombosis (Spaet and Ts'ao, 1969; Puett et al., 1973; Jamieson, 1974). This may be relevant to PSS. Once vascular narrowing is established hypoxia could stimulate further collagen synthesis (Chvapil et al., 1973) and so may perpetuate the disease.

\section{Conclusion}

There is now good evidence that excess collagen formation is an integral feature of PSS. Immunological changes certainly occur in association with this disease and may well be important in its pathogenesis. They may be responsible for vascular narrowing and obstruction, which in turn leads to collagen proliferation and atrophy of tissues. 